Proc. of the 15th Int. Workshop on Slow Positron Beam Techniques and Applications, Prague, September 2-6, 2019

\title{
Hybrid Imaging and Timing Ps Laser Excitation Diagnostics for Pulsed Antihydrogen Production
}

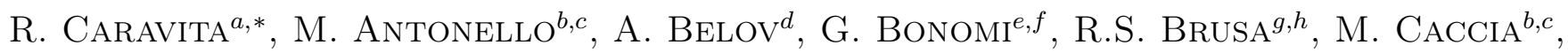
A. Camper ${ }^{a}$, F. Castellit ${ }^{b, i}$, D. Comparat ${ }^{j}$, G. Consolati $^{b, k}$, A. Demetrio ${ }^{l}$,

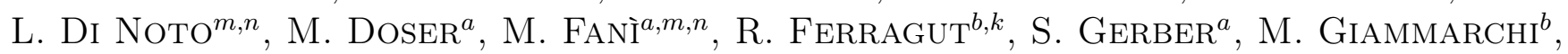
A. Gligorova ${ }^{o}$, L.T. GlöGgler ${ }^{a}$, F. Guatieri ${ }^{g, h}$, S. Haider $^{a}$, A. Hinterberger ${ }^{a}$, O. Khalidova $^{a}$, D. KrasnickÝ ${ }^{n}$, V. Lagomarsino ${ }^{m, n}, \mathrm{C}$ Malbrunot $^{a}$, S. Mariazzi ${ }^{g, h}$,

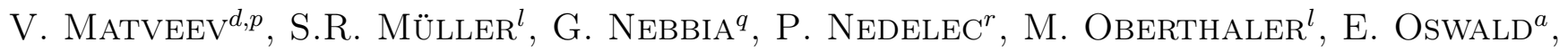

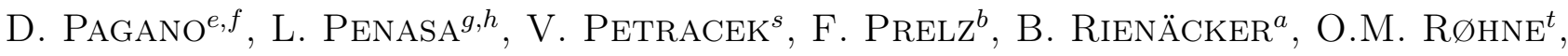

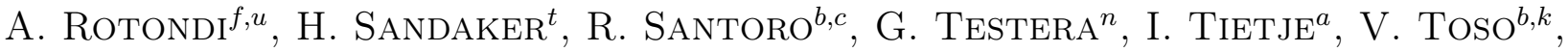

T. WOlZ ${ }^{a}$, C. Zimmer ${ }^{a}$ AND N. ZuRlo ${ }^{f, v}$

${ }^{a}$ Physics Department, CERN, 1211 Geneva 23, Switzerland

${ }^{b}$ INFN Milano, via Celoria 16, 20133, Milano, Italy

${ }^{c}$ Department of Science, University of Insubria, Via Valleggio 11, 22100 Como, Italy

${ }^{d}$ Institute for Nuclear Research of the Russian Academy of Science, Moscow 117312, Russia

${ }^{e}$ Department of Mechanical and Industrial Engineering, University of Brescia, via Branze 38, 25123 Brescia, Italy

${ }^{f}$ INFN Pavia, via Bassi 6, 27100 Pavia, Italy

${ }^{g}$ Department of Physics, University of Trento, via Sommarive 14, 38123 Povo, Trento, Italy

${ }^{h}$ TIFPA/INFN Trento, via Sommarive 14, 38123 Povo, Trento, Italy

${ }^{i}$ Department of Physics, University of Milano, via Celoria 16, 20133 Milano, Italy

${ }^{j}$ Laboratoire Aimé Cotton, Université Paris-Sud, ENS Cachan, CNRS, Université Paris-Saclay, 91405 Orsay Cedex, France

${ }^{k}$ Politecnico of Milano, Piazza Leonardo da Vinci 32, 20133 Milano, Italy

${ }^{l}$ Kirchhoff-Institute for Physics, Heidelberg University, Im Neuenheimer Feld 227, 69120 Heidelberg, Germany

${ }^{m}$ Department of Physics, University of Genova, via Dodecaneso 33, 16146 Genova, Italy

${ }^{n}$ INFN Genova, via Dodecaneso 33, 16146 Genova, Italy

${ }^{\circ}$ Stefan Meyer Institute for Subatomic Physics, Austrian Academy of Sciences,

Boltzmanngasse 3, 1090 Vienna, Austria

${ }^{p}$ Joint Institute for Nuclear Research, 141980 Dubna, Russia

${ }^{q}$ INFN Padova, via Marzolo 8, 35131 Padova, Italy

${ }^{r}$ Institute of Nuclear Physics, CNRS/IN2p3, University of Lyon 1, 69622 Villeurbanne, France

${ }^{s}$ Czech Technical University, Prague, Břehová 7, 11519 Prague 1, Czech Republic

${ }^{t}$ Department of Physics, University of Oslo, Semælands vei 24, 0371 Oslo, Norway

${ }^{u}$ Department of Physics, University of Pavia, via Bassi 6, 27100 Pavia, Italy

${ }^{v}$ Department of Civil Engineering, University of Brescia, via Branze 43, 25123 Brescia, Italy

In this work we present a hybrid detection method providing simultaneous imaging and timing information suitable for fully monitoring positronium (Ps) formation, its laser excitation, and its spatial propagation for the first trials of pulsed antihydrogen $(\overline{\mathrm{H}})$ production through a charge-exchange reaction with trapped antiprotons $(\overline{\mathrm{p}})$. This combined method, based on the synchronous acquisition of an EJ-200 scintillation detector and a microchannel plate (MCP) detector with a dual readout (phosphor screen image and electrical pick-up signal), allows all relevant events in the experiment to be accurately determined in time while allowing high resolution images of $\mathrm{e}^{+}$from Ps laser photodissociations to be acquired. The timing calibration process of the two detectors discussed in details as well as the future perspectives opened by this method.

DOI: 10.12693/APhysPolA.137.96

PACS/topics: 39.10.+j, 36.10.Dr, 78.79.Bj

*corresponding author 


\section{Introduction}

Probing experimentally the gravitational acceleration of neutral antimatter atoms is the main goal of the AEḡIS collaboration based at CERN Antiproton Decelerator [1], aiming to use a Moiré deflectometer [2], combined with high-resolution position-sensitive and timing detectors [3, 4], to detect the time-of-flight and the vertical free-fall displacement of a beam of antihydrogen atoms. Pulsed $\overline{\mathrm{H}}$ production, yet to be experimentally demonstrated, is the key advancement to pave the way to this approach, as it would open the possibility to measure the atoms' time-of-flight, unavailable from currently available trap-based methods $[5,6]$.

Pulsed $\overline{\mathrm{H}}$ production will be realized in AE $\bar{g} I S$ using a charge-exchange reaction between trapped antiprotons $(\overline{\mathrm{p}})$ and laser-excited Rydberg Ps atoms [7, 8]. In synthesis (see Fig. 1a, for a visual reference), a plasma of cold $\bar{p}$ is electro-magnetically confined in a high (1 T) magnetic field region and it is illuminated by a ns- burst of Rydberg Ps. Ps itself is produced above the $\bar{p}$ confinement trap from the conversion of positrons $\left(\mathrm{e}^{+}\right)$in a suitable $\mathrm{e}^{+}$-Ps converter, and then immediately excited to its Rydberg levels with a two-step laser excitation scheme to increase the charge-exchange cross-section.

One of the key advances towards developing the first pulsed $\overline{\mathrm{H}}$ source via charge-exchange with Rydberg Ps consists of developing a fast and reliable detection method for (slow) Ps formation, pulsed two-step laser excitation, and directioning towards the $\overline{\mathrm{p}}$ cloud, compatible with the cryogenic $1 \mathrm{~T}$ magnetic field environment necessary for $\bar{p}$ plasma handling.

(a)

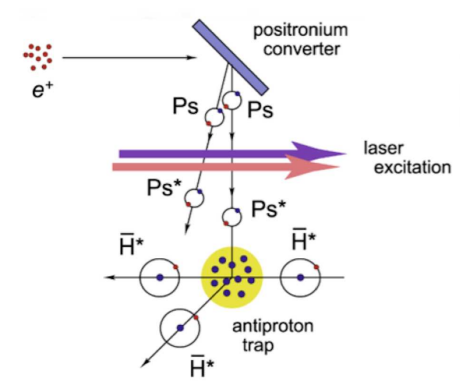

(b)

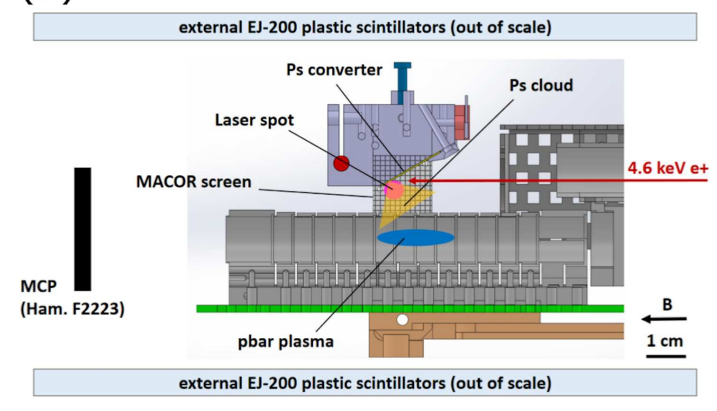

Fig. 1. (a) conceptual scheme of the AEgIS experiment for pulsed antihydrogen production. (b) schematic view of the experimental setup.
These environmental requirements impose stringent limitations to standard techniques widely adopted in the $\mathrm{e}^{+}$community, such as Single-Shot Positron Annihilation Lifetime Spectroscopy (SSPALS) [9], as most scintillating materials and photo-sensors are unsuitable for operating at liquid helium temperature and in a high magnetic field. Yet, the possibility to adapt SSPALS and charged particles MCP imaging to these environmental conditions has been recently shown to be possible thanks to the use of inexpensive plastic scintillators for wide solid angle coverage outside the magnetic field [10] and the efficient detection of photo- $\mathrm{e}^{+}$originating from laser photo-dissociations with efficient light collection in high-vacuum [11, 12].

In this work, we present a fully-hybrid SSPALS + MCP acquisition system combining the effectiveness of SSPALS for properly quantifying the amount of formed and laser-addressed Ps to the high sensitivity of a microchannel plate (MCP) detector to detect photo-e ${ }^{+}$from Ps laser-induced photodissociations [11], providing accurate imaging and timing information of all relevant events in the experimental sequence $\left(\mathrm{e}^{+}\right.$implantation, $\mathrm{e}^{+}$-laser delay, and photo-e ${ }^{+}$detection timing).

\section{Experimental}

The hybrid SSPALS + MCP detection method is based on the simultaneous acquisition of a semi-cylindrical wide-area EJ-200 plastic scintillation detector wrapped around the AEḡIS cryostat (thoroughly described in [10]) and a dual-readout Hamamatsu F2223 MCP/phosphor screen assembly placed in the $\overline{\mathrm{H}}$ production region.

A sketch of the current hardware configuration of the innermost part of the experiment is shown in Fig. 1. A nanoporous silicon positron-Ps converter developed for cryogenic use [13] is located $1.5 \mathrm{~cm}$ above the cylindrical Malmberg-Penning trap for $\bar{p}$ plasmas storage for the pulsed $\overline{\mathrm{H}}$ experiment $[14,15]$. An aperture in the upper part of the trap electrodes is realized to allow Rydberg Ps atoms produced outside to reach the $\bar{p}$ plasma. Ps atoms are produced from $\sim 10 \mathrm{~ns}$ bursts of $\mathrm{e}^{+}$implanted with $4.6 \mathrm{keV}$ energy in the target where they are efficiently converted into Ps atoms and re-emitted into vacuum after cooling by collisions in the material porosities. Two laser pulses are sent from one side to excite the re-emitted Ps cloud first to $n=3(205.045 \mathrm{~nm})$, then to Rydberg levels $(1700 \mathrm{~nm})$ using a two-step laser excitation scheme [16]. Optionally, an intense IR laser pulse at $1064 \mathrm{~nm}$ could be introduced in place of the $1700 \mathrm{~nm}$ laser to selectively photo-ionize $n=3$ Ps and estimate its amount [17].

The EJ-200 plastic scintillator, acquired by two magnetic-shielded EMI 9954B photomultipliers, is able to detect (mainly through Compton scattering) the time distribution of the gamma rays originating from $\mathrm{e}^{+}$and Ps annihilations. Thus, it allows SSPALS spectra to be recorded, even if with a total efficiency smaller than comparable detectors used in room-temperature setups. 
The MCP/phosphor screen assembly is configured with a dual imaging and timing readout. The imaging readout is performed by an ORCA-Flash4 CMOS camera equipped with a vacuum lens system for efficient light collection [12]. The timing readout system digitises the electronic pick-up signal from the MCP phosphor screen via an high-voltage bypass capacitor and the same Teledyne-LeCroy HDO4096 12 bit $1.0 \mathrm{GHz}$ oscilloscope used for digitizing the scintillation detector signal providing a common timebase.

The static electric field in the Ps excitation region can be controlled by biasing the $\overline{\mathrm{p}}$ trap electrodes. A linear voltage ramp from positive $28 \mathrm{~V}$ on the most upstream electrode to positive $20 \mathrm{~V}$ on the electrode $15 \mathrm{~mm}$ after the end of the Ps converter was used to efficiently collect and accelerate the photo-dissociated $\mathrm{e}^{+}$on the front face of the MCP detector. This voltage configuration was optimized to obtain the highest signal-to-noise ratio with the imaging detector, while keeping $-180 \mathrm{~V}$ negative voltage on the front face of the MCP to effectively shield secondary-emitted $\mathrm{e}^{-}$by the diffusion of a fraction of the deep-UV light on the many metal surfaces, undergoing photoelectric effect, and to further accelerate the incoming $\mathrm{e}^{+}$at the end of their $13.9 \mathrm{~cm}$ flight towards the detector to increase their detection efficiency.

\section{Results}

An example measurement obtained by the hybrid SSPALS + MCP detector is shown in Fig. 2. Two series of pulsed Ps production measurements were acquired without the presence of any lasers and with the presence of two $205.045 \mathrm{~nm}$ and $1064 \mathrm{~nm}$ synchronous laser pulses efficiently photoionizing Ps through the excitation to $n=3$ (as in [17]). Each readout chain allows different information to be extracted.

SSPALS spectra can be constructed averaging many EJ-200 scintillator signals (Fig 2a), thus providing a nsaccurate measurement of $\mathrm{e}^{+}$arrival time on the converter and the detection of the $\gamma$-ray distribution originating from $\mathrm{Ps} / \mathrm{e}^{+}$annihilations in-flight and by collisions with matter surfaces. SSPALS is a valuable tool for quantitative estimations of Ps production and laser excitation efficiencies, as described in many previous works $[10,17]$.

The MCP phosphor screen image shows the unambiguous detection of photo-dissociated $\mathrm{e}^{+}$(as in [10-12]) and the presence of a faint background correlated to the presence of the UV laser. This is likely due to the diffusion of a small portion of the UV light from the MACOR screen (shown in Fig. 1), used to align the laser beams, to the MCP front face through specular reflections on the reflecting metal surfaces inside the experiment (Fig. 2c). UV photons at $205 \mathrm{~nm}$ can indeed be detected efficiently by the Hamamatsu F2223 due to absorption/photoelectric effect on its front face, initiating spurious electron cascades in the channels. Secondary electrons backgrounds from other external sources are shielded by the $-180 \mathrm{~V}$ bias voltage used to accelerate photo-e $\mathrm{e}^{+}$.
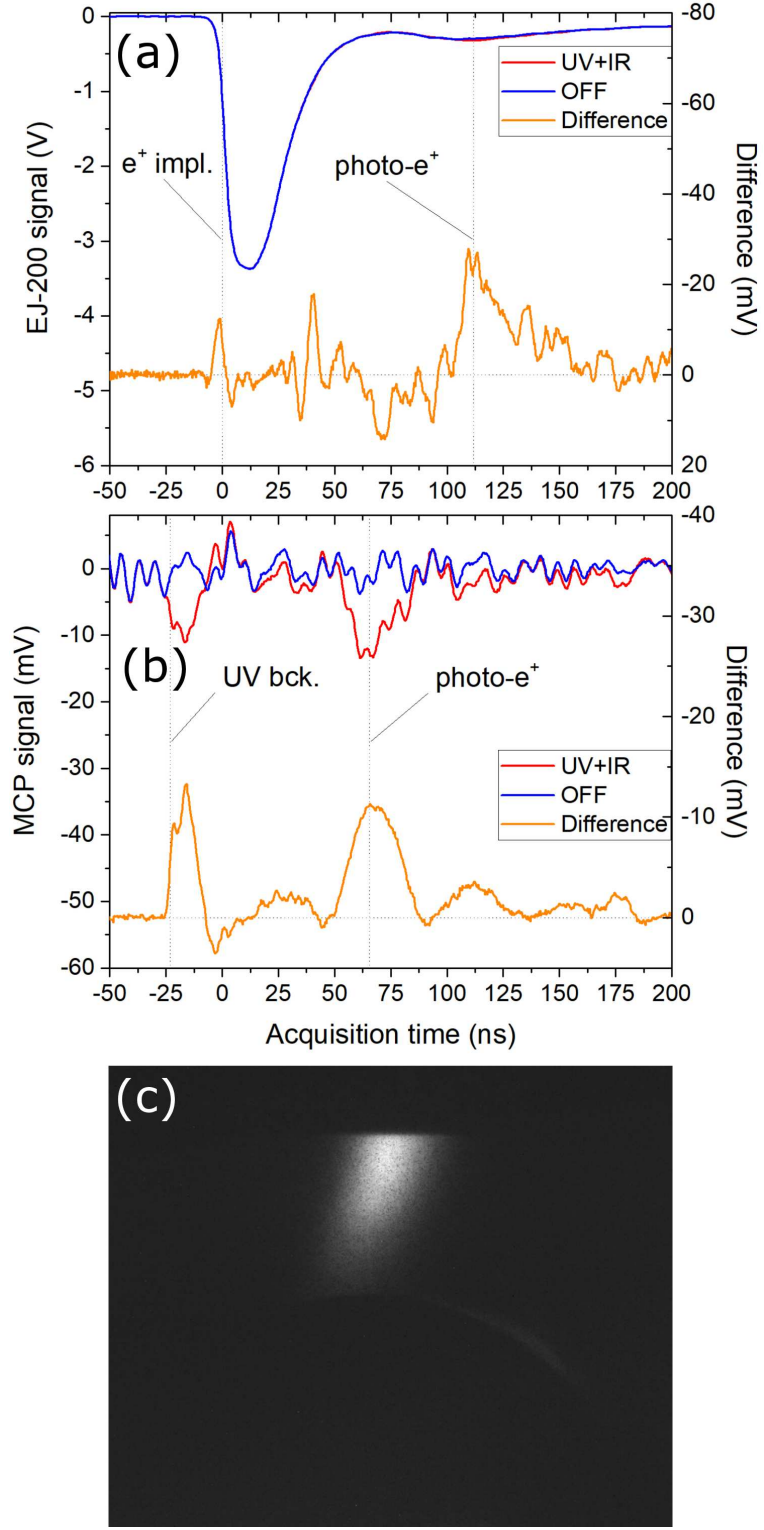

Fig. 2. Overview of the hybrid timing- and positionsensitive MCP+SSPALS detection scheme, showing the simultaneously-acquired data for the reference timing calibration measurement discussed in the text. Difference curves (orange) are obtained subtracting the average waveforms obtained with two laser pulses efficiently photo-ionizing Ps (red) to the average waveforms without any laser (blue).

The electrical MCP phosphor screen signal shows two peaks at $\sim 80$ ns distant from each other (see Fig. $2 b$ ), which can be related to the presence of the UV-induced background observed in the image and the photo- $\mathrm{e}^{+}$detection peak. This interpretation is supported by the disappearance of the second peak when the strong IR ionizing laser is absent, whereas the presence of first peak is correlated to the presence of the UV laser alone and its position in time shifts accoding to its set relative delay (see Fig. 3). 


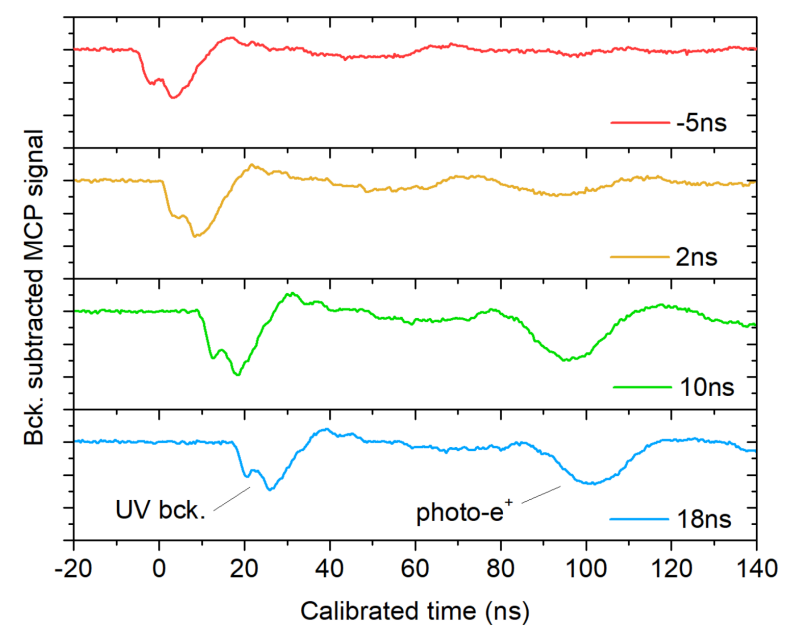

Fig. 3. Single-shot time-resolved detection of photo-e ${ }^{+}$ for accurate $\mathrm{e}^{+}$- laser timing measurements for different $\mathrm{e}^{+}$-laser delays set by a timing FPGA used for fine synchronization. The rigid time shift of both the UV laser background peak (left) and the photo-e ${ }^{+}$peak (right) is clearly visible. The UV laser background peak is suitable for self-calibrating the photo- $\mathrm{e}^{+}$arrival time independently of the set $\mathrm{e}^{+}$-laser delay.

\subsection{Timing calibration}

The information of the EJ-200 SSPALS spectrum and the MCP phosphor screen electric signal can be combined to provide an accurate timing measurement of all the relevant events happening in the experiment in the proximity of the $\overline{\mathrm{H}}$ production instant (the $\mathrm{e}^{+}$implantation time, the UV laser arrival time in the experiment and the time of the photo- $\mathrm{e}^{+}$detection), provided that a calibration of the two detector timebases is performed to compensate for the different signal transit times through their respective acquisition chains $\left(\Delta t_{\text {calib }}\right)$.

A suitable calibration can be obtained by the measurement shown in Fig. 2 observing that the photo-e ${ }^{+}$peak has to be detected simultaneously (in a first approximation) by both detectors. More precisely, the photo- $\mathrm{e}^{+}$ peak on the EJ-200 scintillator has to be delayed, with respect to that detected by the MCP, by the time-offlight of the $511 \mathrm{keV}$ gamma rays from their annihilation point on the MCP surface to the scintillator $(2.1 \mathrm{~ns})$.

The accuracy of this calibration is set mainly by the broadening of the photo- $\mathrm{e}^{+}$time distributions due to the $\mathrm{e}^{+}$burst length $(\sim 15-20 \mathrm{~ns}$ full-width at half maximum (FWHM) ) according to the beam intensity, higher than the time resolution of both detectors $(\sim$ a few ns). The criterium used to calibrate the two timebases was to synchronize the two peak values accounting for the $2.1 \mathrm{~ns}$ gamma rays time-of-flight. The peak time was measured fitting a gaussian function to the difference data of Fig. 2 in a window of 3 ns FWHM around the peak value. By this method, it was determined that the two photo- $\mathrm{e}^{+}$peaks were observed, in the acquisition timebase, at $t_{\mathrm{MCP}}=68.2 \pm 0.2 \mathrm{~ns}$ and $t_{\mathrm{EJ}-200}=115.3 \pm 1.8 \mathrm{~ns}$.
The error bars are determined by varying the choice of the (otherwise arbitrary) fitting window by some tens of ns. It is convenient, to define a common timebase for both detectors, to calibrate the timing of the digitized MCP signal with respect to that of the EJ-200, since the $\mathrm{e}^{+}$implantation is the first event in a typical experimental sequence and it is practical to refer all other times to it. Thus, calibrated MCP time can be defined as $t^{\prime}=t+\Delta t_{\text {calib }}$ with

$\Delta t_{\text {calib }}=t_{\mathrm{EJ}-200}-t_{\mathrm{MCP}}-2.1 \mathrm{~ns}=(45.0 \pm 1.8) \mathrm{ns}$.

One can independently verify the goodness of this calibration by varying the time at which the laser is shot on the Ps sample (the UV laser is only $1.5 \mathrm{~ns}$ long) and verifying the consistent appearance/disappearance of the photo- $\mathrm{e}^{+}$peak as a function of the (measured) delay of the laser peak. Example results are shown in Fig. 3, showing the ability of the MCP digited signal in monitoring the laser position and showing the disappearance/appearance of the photo- $\mathrm{e}^{+}$peak when the $\mathrm{e}^{+}-$ laser delay is positive with respect to the $\mathrm{e}^{+}$implantation instant.

\subsection{Photo-e $e^{+}$time-of-flight}

The MCP phosphor screen pick-up signal offers the possibility to measure accurately the photo- $\mathrm{e}^{+}$ time-of-flight by measuring self-consistently the delay time between the UV laser arrival and the photo-e ${ }^{+}$ peak. Indeed, the measured $(83.7 \pm 1.8)$ ns delay time (see Fig. 2b) is in very good agreement with a simple analytical calculation of the expected photo- $\mathrm{e}^{+}$ time-of-flight

$$
\Delta t_{\mathrm{TOF}}=\sqrt{\frac{2 m}{q E_{0}}} \Delta z_{1}+\sqrt{\frac{m}{2 q V_{0}}} \Delta z_{2} \approx 82 \mathrm{~ns} .
$$

This formula was obtained by modelling the photo-e ${ }^{+}$ flight in three parts: an initial uniform acceleration of the formed photo- $\mathrm{e}^{+}$by the linear potential ramp used to collect them $\left(E_{0}=200 \mathrm{~V} / \mathrm{m}\right.$ for $\Delta z_{1}=15 \mathrm{~mm}$ distance); the subsequent fast acceleration by the following electrodes set at ground potential, quickly raising the $\mathrm{e}^{+}$ axial kinetic energy to $V_{0}=20 \mathrm{~V}$; the final free-flight (with a constant axial velocity) for a $\Delta z_{2}=139.0 \mathrm{~mm}$ distance. The final acceleration by $-180 \mathrm{~V}$ in the proximity of the MCP detector was not modelled as it introduces only a negligible $(\approx \mathrm{ns})$ constant shift to the total time-of-flight.

\section{Conclusions}

In this work, we presented a fully-hybridized SSPALS + MCP detector retaining all characteristics of both individual methods while bridging between the space-integrated time-explicit information of SSPALS spectra and the time-integrated space-explicit information of MCP images through the synchronous acquisition of the electric pick-up signal of the MCP phosphor screen. This extra acquisition channel, provided a timing calibration with a commonly observed pulsed signal, 
allows the UV laser to be monitored (both in time and intensity), the accurate determination of its delay with respect to the $\mathrm{e}^{+}$implantation instant, and provides a self-referenced time-resolved method to detect photo-e ${ }^{+}$ from Ps photodissociations, unaffected by the UV light background, which could be used in future experiments to conduct time-resolved time-of-flight spectroscopy of Ps energy levels in a strong magnetic field.

Finally, it will provide the full real-time monitoring of $\mathrm{e}^{+}$and laser conditions to perform the first trials of pulsed $\overline{\mathrm{H}}$ formation in AE $\overline{\mathrm{g} I S}$, for which the time is now ripe.

\section{References}

[1] M. Doser, et al. (AEgIS collaboration), Class. Quant. Grav. 29, 183009 (2012).

[2] S. Aghion, et al. (AEgIS collaboration), Nat. Commun. 5, 4538 (2014).

[3] S. Aghion, et al. (AEgIS collaboration), J. Instrum. 4, 082 (2013).

[4] S. Aghion, et al. (AEgIS collaboration), J. Instrum. 9, P06020 (2014).

[5] M. Amoretti, C. Amsler, G. Bonomi, et al., Nature 419, 456 (2002).
[6] G.B. Andersen et al., Nature Physics 7, 558 (2011).

[7] C.H. Storry et al., (ATRAP collaboration), Phys. Rev. Lett. 93, 263401 (2004).

[8] D. Krasnický, R. Caravita, C. Canali, G. Testera, Phys. Rev. A 94, 022714 (2016).

[9] D.B. Cassidy, Eur. Phys. J. D 72, 53 (2018).

[10] R. Caravita et al. (AEgIS collaboration), AIP Conf. Proc. 2182, 030002 (2019).

[11] A. Camper et al. (AEgIS collaboration), EPJ Web Conf., 198, 00004 (2019).

[12] C. Amsler et al. (AEgIS collaboration), Nucl. Instr. Meth. Phys. Res. B 457, 44 (2019).

[13] S. Mariazzi, P. Bettoti, S. Larcheri, L. Toniutti, R.S. Brusa, Phys. Rev. B 81, 235418 (2010).

[14] D. Krasnický et al. (AEgIS collaboration), AIP Conf. Proc. 1521, 144 (2013).

[15] S. Aghion et al. (AEgIS collaboration), Eur. Phys. J. D 72, 76 (2018).

[16] S. Cialdi, I. Boscolo, F. Castelli, F. Villa, G. Ferrari, M.G. Giammarchi, Nucl. Instrum. Meth. Phys. Res. B 269, 1527 (2011).

[17] S. Aghion et al. (AEgIS collaboration), Phys. Rev. A 94, 012507 (2016). 